\title{
Are Immunohistochemical Markers Useful in Phenotypic Gastric Cancer Classification?
}

\author{
Federica Di Pinto $^{a} \quad$ Raffaele Armentano ${ }^{a} \quad$ Graziana Arborea $^{a} \quad$ Nicolò Schena $^{a}$ \\ Rossella Donghiab Anna Maria Valentini ${ }^{\mathrm{a}}$ \\ aDepartment of Pathology, National Institute of Gastroenterology "S. de Bellis," Research Hospital, Castellana \\ Grotte, Bari, Italy; bUnit of Epidemiological Research on Frailty Phenotype, National Institute of Gastroenterology \\ "S. de Bellis," Research Hospital, Castellana Grotte, Bari, Italy
}

\section{Keywords}

MLH1 · p53 · HER2 - E-cadherin - Epstein-Barr virus

\begin{abstract}
To identify useful markers for prognostic and therapeutic purposes, The Cancer Genome Atlas (TCGA) provided a molecular classification of gastric cancers (GCs). Previous studies have used immunohistochemistry (IHC) and chromogenic in situ hybridization (CISH) to define immunophenotypic surrogate markers of the molecular alterations. Some critical issues concerning the correct definition of immunophenotypic groups have emerged in these studies that employed tissue microarrays (TMAs). We performed an immunophenotypic classification by evaluating MLH1, p53, HER2, E-cadherin, and Epstein-Barr virus (EBV) on the whole section of the surgical GC samples compared to most of the studies conducted on TMAs. We also investigated the immunohistochemical expression of PD-L1, a known therapeutic target. We identified the following immunophenotypic groups: EBV (2.9\%); mismatch repair deficient (MMR-D) (7.2\%); overexpressed p53 and/or HER2+ (61.4\%); aberrant E-cadherin (11.4\%); and normal pattern (17.1\%). The use of surgical samples emphasized that some immunohistochemical markers
\end{abstract}

were not useful for properly classifying the GC specimens. We can state that EBV (significantly correlated to PD-L1 expression) and MMR-D GCs are well-defined groups, mutually exclusive, and easily assessable with $\mathrm{IHC}$ and $\mathrm{CISH}$, and could be candidates for immunotherapy with PD-1/PD-L1 inhibitors. As regards $\mathrm{p} 53$, our findings suggest that IHC assessment may be responsible for a misclassification of GC groups. Immunohistochemical evaluation of E-cadherin needs to be standardized, particularly in terms of the heterogeneous cytoplasmic/membranous staining pattern. Whether to consider the normal-pattern group as a separate category remains to be clarified. Because GC specimens with known therapeutic targets account for only $40 \%$, we suggest reviewing the immunophenotypic classification to find new therapeutic targets, such as PD-L1, MLH1, and HER2.

(c) 2020 S. Karger AG, Base

\section{Introduction}

Understanding the heterogeneous molecular characteristics of gastric cancer (GC) is critical in view of the practical implications for diagnostic assessment and prognostic evaluation and to develop new therapeutic karger@karger.com

www.karger.com/ocl

Karger
(C) 2020 S. Karger AG, Basel

(n)
Anna Maria Valentini

Department of Pathology, National Institute of Gastroenterology "S. de Bellis" Research Hospital, Castellana Grotte, via Turi 27

IT-70013 Castellana Grotte, Bari (Italy)

am.valentini@irccsdebellis.it 
strategies. GC heterogeneity is a relevant issue and encompasses intertumoral and intratumoral heterogeneity (both spatial and temporal) [1]. Most patients are diagnosed with advanced disease when the outcome is poor, with a 5 -year survival rate of $<40 \%$, even in multimodal treatment settings. The main reasons for these disappointing results are tumor aggressiveness and therapy resistance $[2,3]$.

Molecular targeted therapies available for advanced GC are limited to trastuzumab in patients with HER2 (human epidermal growth factor receptor 2)-positive tumors [4], and ramucirumab (anti-VEGFR2) as secondline treatment $[5,6]$. In 2017, the FDA approved pembrolizumab (anti-PD-1) for the treatment of cancer patients with high microsatellite instability (MSI-H), and it has been approved as third-line treatment for GCs expressing PD-L1 [7].

The latest advances in molecular methods have increased our understanding of GC biology, and led to the development of a new comprehensive molecular classification [8]. To study the characteristics of GC and to identify markers that can be useful for prognostic and therapeutic purposes, in 2014, The Cancer Genome Atlas (TCGA) provided a molecular classification of GCs, subdivided into four subtypes, based on the presence of Epstein-Barr virus (EBV), MSI, genomic stability (GS), and chromosomal instability (CIN) [8].

The complex molecular methodologies used in this molecular classification cannot be widely used for routine clinical diagnostics in pathology laboratories, whereas since 2016 some studies have relied on immunohistochemistry (IHC) and chromogenic in situ hybridization (CISH) to define potential surrogate markers of the molecular alterations [9-13]. Indeed, immunophenotypic classifications use some markers deriving from the TCGA molecular classification, such as: EBV (for the EBV subtype), MLH1 (for the MSI subtype), p53 (for the CIN subtype), and epithelial cadherin (E-cadherin) (for the GS subtype).

Studies on immunophenotypic classification are still ongoing, because ever since the first study by Setia et al. [10], they have highlighted some critical issues concerning the correct immunohistochemical definition of the subtypes, especially for p53 and E-cadherin assessments [14-17].

We carried out an immunophenotypic classification using IHC and CISH for the assessment of MLH1, p53, HER2, E-cadherin, and EBV on the whole section of surgical GC specimens, whereas most of the studies were performed on tissue microarrays (TMAs). The limited amount of TMA tissue could underestimate the expression of tissue markers. Moreover, because TCGA data showed that HER2 amplification was most commonly present in CIN tumors, to better identify this group with immunohistochemical staining, we also determined the HER2 status. In addition, we investigated the immunohistochemical expression of PD-L1, a well-known marker for immune checkpoint inhibitor therapy, in the different immunophenotypic subtypes.

\section{Subjects and Methods}

\section{Patients and Tumor Characteristics}

Between 2014 and 2017, a total of 46 males and 24 females (aged 34-83 years, median 65.8) who underwent curative gastrectomy for primary GC at the National Institute of Gastroenterology "S. de Bellis," Castellana Grotte, Bari, Italy, were included in our study. The inclusion criterion was not having undergone neoadjuvant therapy. For each tumor, the histological type was reevaluated from hematoxylin-eosin (HE) slides. Age, sex, side, and pT and pN status were obtained from pathology reports and HE slide review. The tumor site was proximal (cardia, corpus, and fundus) in 37 patients and distal (antrum/pylorus) in 33 patients. According to the Laurén classification, the histological types of the 70 GCs were 36 diffuse and 34 intestinal. Tissue samples were chosen to include a component of both normal and tumor cells. The pathological and clinical features of the patients are shown in Table 1.

\section{Immunohistochemical Staining}

IHC was performed on formalin-fixed paraffin-embedded sections ( $4 \mu \mathrm{m}$ thick). MLH1 (MAb ES05, diluted 1:50; Agilent Technologies, Santa Clara, CA, USA), p53 (MAb DO7, diluted 1:500; A. Menarini Diagnostics, Florence, Italy), E-cadherin (MAb NCH38, diluted 1:100; Agilent Technologies), and HER2 (Polyclonal Antibody; Agilent Technologies) were studied. IHC for PD-L1 (clone E1L3N; Cell Signaling Technology, Danvers, MA, USA) was performed as previously described [18]. IHC was analyzed on a fully automated IHC stainer (Bond III; Leica Biosystems, Buccinasco, Italy). All antibodies were diluted in Bond Primary Antibody diluents (Leica Biosystems). Sections were retrieved in Epitope Retrieval Solution 2 (High pH) (Leica Biosystems) for MLH1, p53, E-cadherin, and PD-L1, and in Epitope Retrieval Solution 1 (Low pH) (Leica Biosystems) for HER2. Monoclonal antibodies against PD-L1, MLH1, p53, and E-cadherin and polyclonal antibody anti-HER2 protein were applied to the tissue sections and replaced with PBS on negative control slides. A Bond Polymer Refine Detection Kit (Leica Biosystems) was used as the visualization reagent and chromogen, according to the manufacturer's instructions.

PD-L1 immunoreactivity was evaluated on tumor cells, and PD-L1 positivity was defined by the presence of at least $5 \%$ positive cells with membrane staining of any intensity [19]. Cytoplasmic staining was not considered in this study. Tumors with a complete loss of MLH1 staining in tumor cell nuclei were defined as mismatch repair deficient (MMR-D), while tumors with retained nuclear staining were deemed mismatch repair proficient (MMR-P). 
Table 1. Correlation between immunophenotypic classification and clinicopathological features

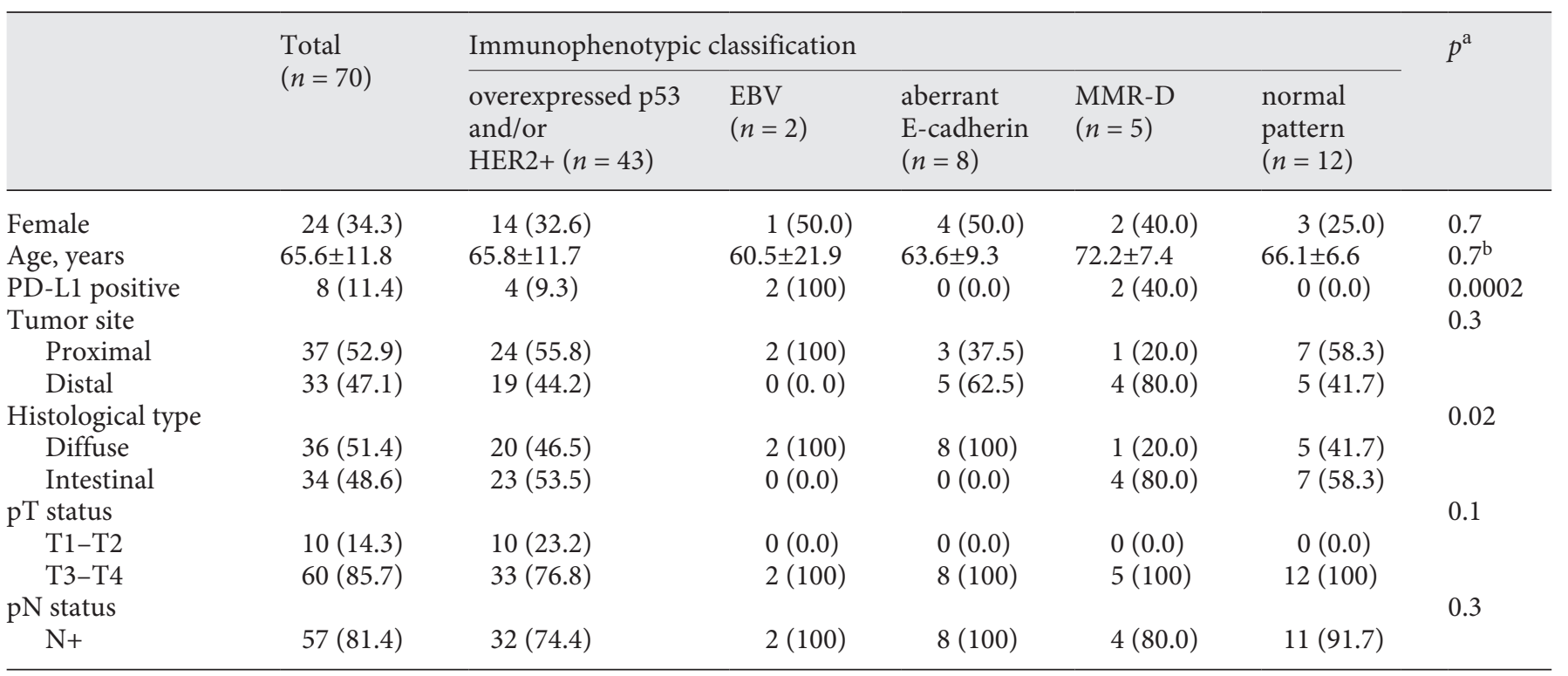

Values are presented as $n(\%)$ or mean \pm SD. Bold type denotes significance. ${ }^{a}$ Fisher's exact test. ${ }^{\mathrm{b}}$ Kruskal-Wallis rank test.

p53 immunoreactivity was evaluated on tumor cells, and only strong staining in $70 \%$ or more of the cell nuclei was interpreted as "overexpression" according to Ando et al. [14]. Staining in less than $70 \%$ of the cell nuclei and completely negative staining were interpreted as "wild type" [14].

For E-cadherin, cases with complete loss of membranous reactivity or only faint cytoplasmic reaction of cells were considered to have aberrant E-cadherin expression [10]. Cases with a membrane staining pattern or a heterogeneous cytoplasmic/membranous staining pattern were considered to show normal E-cadherin expression [10]. When evaluating HER2 scoring, the criteria based on the report by Hofmann et al. [20] were adopted.

\section{HER2 Chromogenic in situ Hybridization}

Only IHC score $2+$ cases were subjected to CISH for the evaluation of HER2 gene amplification. The CISH method was carried out using the ZytoDot 2C SPEC ERBB2/CEN 17 Probe Kit (ZytoVision, Bremerhaven, Germany). The sections (2-3 $\mu \mathrm{m}$ thick) were deparaffinized, incubated in pretreatment buffer for $15 \mathrm{~min}$ at $98-100^{\circ} \mathrm{C}$, and washed with distilled water. After enzymatic digestion for $15 \mathrm{~min}$ at room temperature, the sections were dehydrated and $15 \mu \mathrm{L}$ of HER2 probe was applied. To prevent evaporation during incubation, standard coverslips and rubber cement were used. The sections were subjected to denaturation at $80^{\circ} \mathrm{C}$ for $5 \mathrm{~min}$ and to hybridization at $37^{\circ} \mathrm{C}$ overnight in the ThermoBrite Hybridizer (StatSpin, Norwood, MA, USA). Subsequently, after removing the coverslips, the slides were washed in stringent wash buffer for $5 \mathrm{~min}$ at $75^{\circ} \mathrm{C}$.

HER2 gene amplification was detected by sequential incubation with Anti-DIG/DNP-Mix, HRP/AP-Polymer-Mix, AP-Red Solution, and HRP-Green Solution. Finally, the slides were counterstained with hematoxylin. Sections of a breast cancer known to be HER2 amplified were included as positive controls in each run.
The CISH signal was evaluated using a light microscope $(\times 40$ objective lens) after reviewing the $\mathrm{HE}$ - and IHC-stained slides to identify those areas of invasive GC with the strongest intensity of HER2 expression.

The College of American Pathologists guidelines recommend scoring of at least 20 non-overlapping tumor cell nuclei to calculate the ratio of HER2 to CEN17 signals. HER2 amplification is defined as a HER2/CEN17 ratio $\geq 2$. If this ratio is $<2$, an average HER2 signal per cell count of $<4$ signals indicates no HER 2 amplification, while an average of $>6$ signals indicates HER2 amplification. An average between $\geq 4$ and $<6$ indicates an equivocal result. In the last case, testing IHC or CISH on a different patient sample is recommended [21]. For HER2 status evaluation, the IHC 3+ and IHC 2+ (with gene amplification) cases were regarded as HER2 positive, and the remaining cases as HER2 negative.

\section{EBV Status}

CISH for EBV-encoded RNA (EBER) was performed using an RNAscope detection kit according to the protocol provided by Advanced Cell Diagnostics (Hayward, CA, USA). The sections ( $4 \mu \mathrm{m}$ thick) were baked at $60^{\circ} \mathrm{C}$ for $1 \mathrm{~h}$ and then deparaffinized using the protocol proposed by the manufacturer. The deparaffinized slides were boiled for $10 \mathrm{~min}$ in $\mathrm{pH} 6.0$ citric buffer for antigen retrieval. The slides were further permeabilized by protease treatment at $40^{\circ} \mathrm{C}$ for $30 \mathrm{~min}$ in a HybEZ hybridization oven (Advanced Cell Diagnostics), followed by hybridization with the target probe, amplification, and detection by adding Amp1-4.

Chromogenic detection was performed using DAB followed by counterstaining with hematoxylin. The kit included slides with HeLa cultured cells useable as positive or negative controls depending on the probes adopted for hybridization. Samples resulting brown-stained in any tumor cell nuclei were considered positive. 


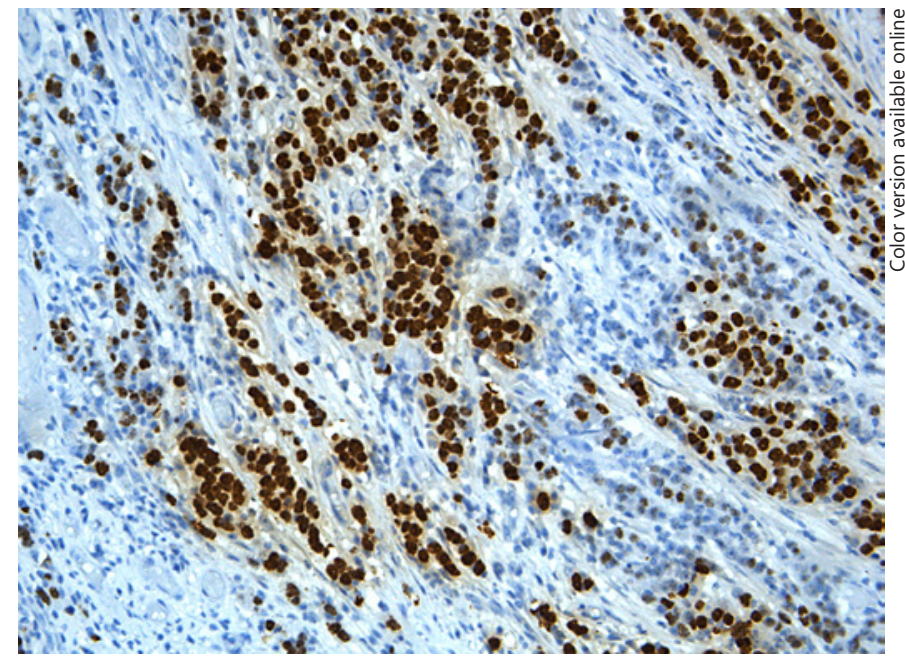

Fig. 1. Epstein-Barr virus chromogenic in situ hybridization shows positive nuclei in the gastric cancer cells, which are surrounded by infiltrating lymphocytes.

\section{Statistical Analysis}

Mean and standard deviation for continuous variables and relative frequency (\%) were used as indices of centrality and dispersion of the distribution for categorical variables. For associations between the categorical variables, we used the $\chi^{2}$ test or Fisher's exact test, depending on the number of observations. For those variables that, when measured on a continuous scale, were not normally distributed, the Wilcoxon rank-sum test (Mann-Whitney and Kruskal-Wallis rank test) was used. When testing the null hypothesis of no association, the probability level of an a error, twotailed, was 0.05. All statistical computations were made using STATA 12.1 Statistical Software (StataCorp) 2014, release 12 (College Station, TX, USA).

\section{Results}

Based on the results of IHC and CISH, we divided our 70 GC cases into the following five groups: EBV, 2 cases; MMR-D, 5 cases; overexpressed p53 and/or HER2+, 43 cases; aberrant E-cadherin, 8 cases; and normal pattern, 12 cases negative for all markers.

Unlike the MLH1 loss and EBV infection patterns, which turned out to be mutually exclusive in some samples, some overlaps regarding p53 overexpression, HER2 positivity, and aberrant E-cadherin stainings were highlighted. We identified 6 samples stained as follows: 2 cases with both aberrant E-cadherin and overexpressing p53, 3 cases with aberrant E-cadherin and HER2 positivity, and 1 case with overexpression of $\mathrm{p} 53$, aberrant E-cadherin, and HER2-positive staining. As

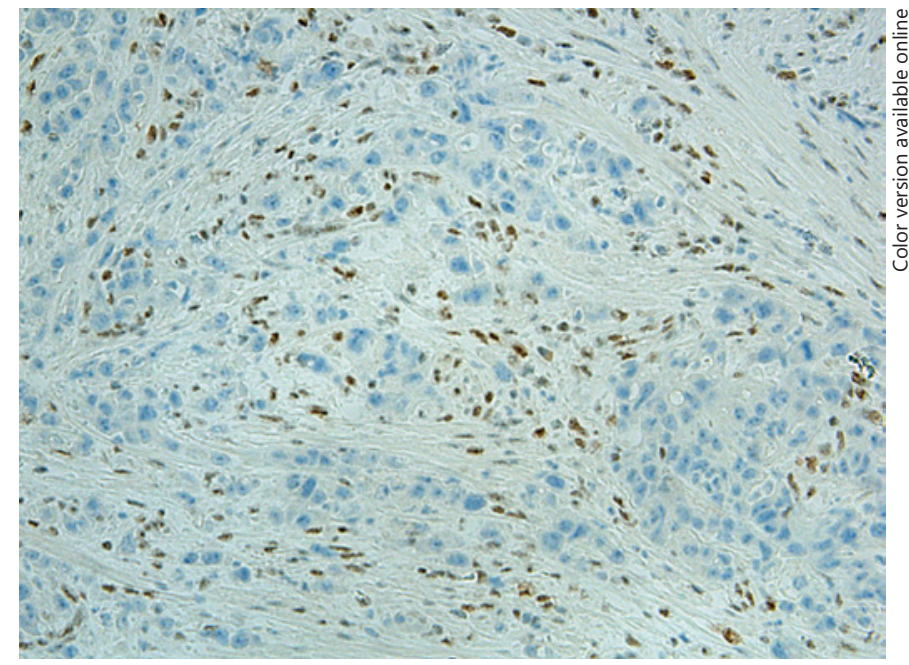

Fig. 2. MLH1 loss in neoplastic cells and retainment in stromal ones (positive internal control).

these specimens were all strongly positive for p53 staining in more than $70 \%$ of the cell nuclei and/or showed HER2 positivity, the aberrant E-cadherin pattern was no longer taken into account, and they were all considered to belong to the group "p53 overexpressed and/or HER2+." The "p53 overexpressed and/or HER2+" group encompassed 24 p53-overexpressing/HER2-negative, 13 p53-/HER2+, and 6 p53-overexpressing/ HER2+ specimens.

Our immunophenotypic classification was significantly correlated with histological type and PD-L1 immunohistochemical expression $(p<0.05)$. Relevant clinicopathological data are summarized in Table 1.

EBV-positive cases $(2.9 \%)$ showed intense nuclear staining in $90 \%$ of the tumoral cells aggregated in cords, clearly surrounded by EBV-negative lymphocytes (Fig. 1). None of the cases showed positivity for EBV in the nontumoral mucosa. Both positive samples were diffuse-type GCs and PD-L1 positive on tumoral cells $(p<0.05)$ (Table 2).

MLH1 loss (Fig. 2) was observed in $7.2 \%$ of the samples. Two (40\%) of the 5 MMR-D GCs were PD-L1 positive, versus $6(9.2 \%)$ of the 65 MMR-P GCs $(p<0.05)$. Four $(80 \%)$ of the 5 MMR-D GCs were distal and intestinal (Table 2).

The "overexpressed p53 and/or HER2+" group included $46.5 \%$ (20 samples) and 53.5\% (23 samples) diffuse- and intestinal-type GCs, respectively (Fig. 3). Among the GCs belonging to this category, 23.3\% were 


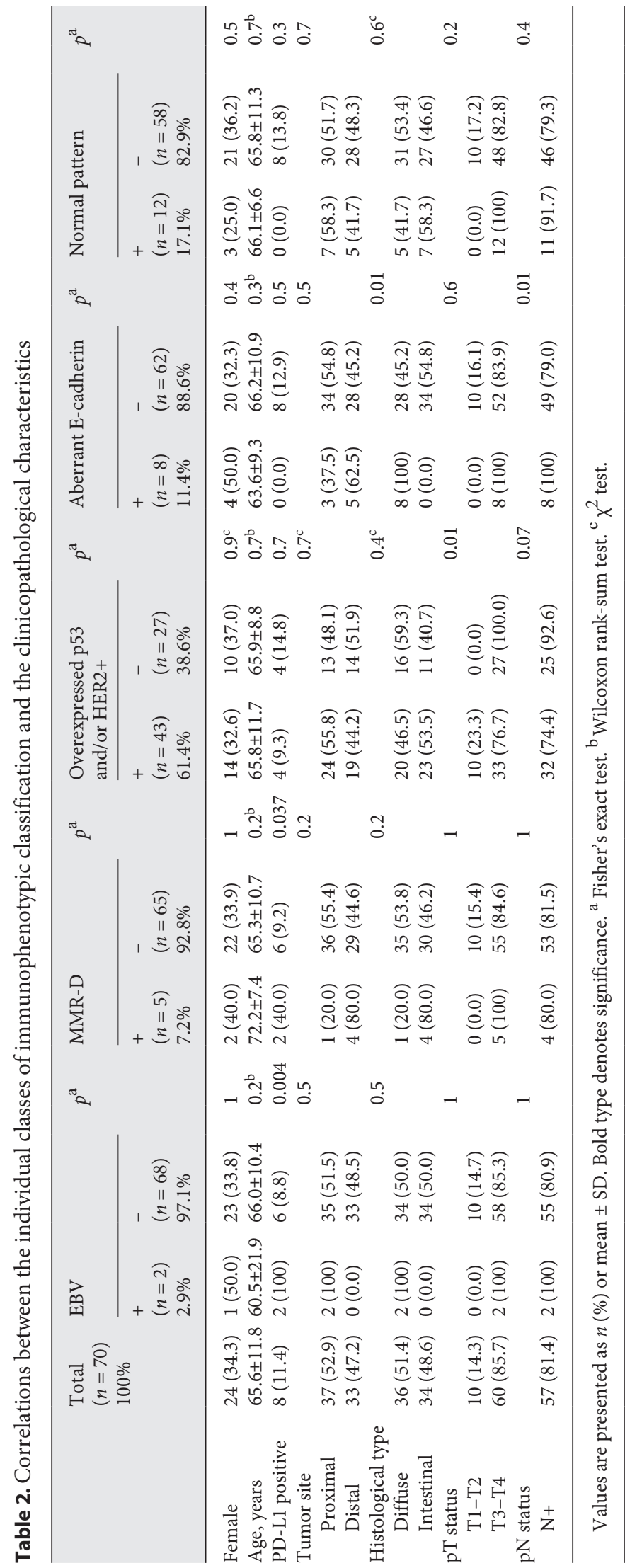

T1-T2 $(p<0.05)$, versus $0 \%$ of the remaining cases (Table 2).

The "aberrant E-cadherin" group included $11.4 \%$ of the samples; all were of the diffuse type $(p<0.05), \mathrm{T} 3-\mathrm{T} 4$, and had a positive $\mathrm{pN}$ status $(p<0.05)$ (Table 2$)$. The "normal pattern" group comprised 5 diffuse-type and 7 intestinal-type GCs. All samples were PD-L1 negative and T3-T4, and 11 (91.7\%) had a positive $\mathrm{pN}$ status (Table 2).

We found that the diffuse GC type was present in more immunophenotypic groups than the intestinal type. Indeed, none of the intestinal-type tumors belonged to the EBV and "aberrant E-cadherin" groups. The "overexpressed p53 and/or HER2+" group included the most cases, both of diffuse-type (55.6\%) and of intestinal-type GCs (67.6\%). The second most widespread groups for the diffuse and intestinal types were the "aberrant E-cadherin" (22.2\%) and the "normal pattern" (20.6\%) group, respectively.

\section{Discussion and Conclusion}

To our knowledge, this is the first study to carry out an immunophenotypic GC classification exclusively based on surgical specimens. Most previous studies were performed on TMAs, which are known not to be representative of intratumor heterogeneous protein expression [22]. Based on the results of IHC and CISH, we found the following five GC groups: EBV (2.9\%); MMR-D (7.2\%); overexpressed p53 and/or HER2+ (61.4\%); aberrant Ecadherin (11.4\%); and normal pattern (17.1\%).

EBV was detected only in lymphoepithelioma-like samples and in the tumor cell compartment, not in lymphocytes and stromal cells, which is consistent with previous study findings. As previously demonstrated [23], we found a significant correlation between EBV infection and PD-L1 expression, suggesting that these tumors are a potential target for anti-PD-1/PD-L1 immunotherapy. EBV infection is easily assessed by CISH, because the nuclei of infected cells appear strongly stained on the sections; therefore, we can state that CISH correctly reflects the molecular status.

In agreement with Birkman et al. [12] and Ahn et al. [13], we found 7.2\% MMR-D GCs. The current study evaluated the MMR status only on MLH1 protein assessment, because it is lost in $>90 \%$ of GCs with MSI [24]. As reported in the literature [23], there was a significant correlation between MLH1 loss and PD-L1 overexpression, so these tumors can also be considered potential targets 


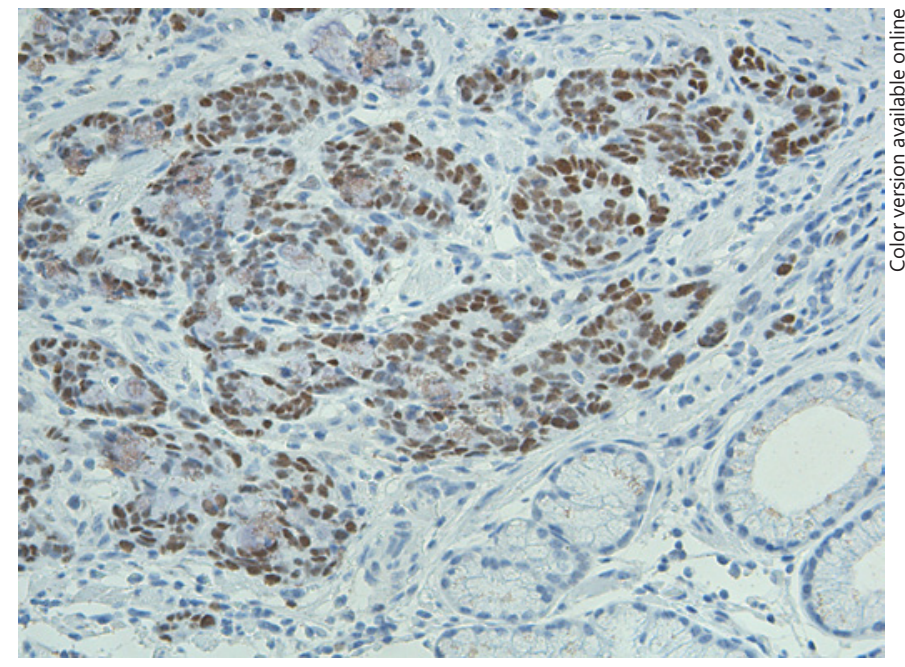

Fig. 3. p53-positive staining in the intestinal gastric cancer type. Note the negative staining in the normal epithelium.

for anti-PD-1/PD-L1 immunotherapy. Immunohistochemical MLH1 evaluation is reliable, because tumors with complete loss of MLH1 staining in tumor cell nuclei, but with a positive internal control, were easily identified as MMR-D, while tumors with few stained nuclei were deemed MMR-P. In fact, the 5 cases with MLH1 loss were subsequently assayed with the molecular Idylla MSI test (Idylla; Biocartis), and they all showed MSI.

The most frequently mutated gene in tumors is p53. In the past, positive p53 immunostaining (cutoff 5-10\%) was considered to be related to p53 gene mutations [25, 26]. Immunohistochemical staining of p53 is a cheap and simple method to indirectly detect gene alterations. In fact, in normal tissues, $\mathrm{p} 53$ protein is unstable and so cannot be detected by immunostaining [27]. In contrast, following a gene mutation, p53 is overexpressed and the cell nucleus results immunostained.

More recently, immunohistochemical p53 assessment has changed. According to Ando et al. [14], immunohistochemically p53-positive tumors show two patterns: aberrant and scattered. The aberrant pattern has widespread staining of $70 \%$ or more of the nuclei of a sample, whereas with the scattered pattern, $20-50 \%$ of the nuclei are stained. Interestingly, making a comparison between molecular and immunohistochemical methods, the authors found that all samples with p53 missense alterations showed an aberrant staining pattern, while all scatteredpattern tumors had the p53 gene wild type. They also highlighted an aberrant p53 pattern in GCs with the wild- type gene [14]. Indeed Hall and Lane [28] reported that there may be 533 overexpression in response to spontaneous genetic errors occurring at a higher frequency in tumors than in normal surrounding tissue. Ando et al. [14] found that $80 \%$ of the samples staining p53 negative ( $0-10 \%$ of the cell nuclei) corresponded to the wild-type gene, and the remaining cases had nonsense and missense mutations.

In agreement with Ando et al. [14], also Cole et al. [29] stated that p53 missense mutations are responsible for p53 overexpression ( $\geq 70 \%$ of stained nuclei). However, unlike Ando et al. [14], Lax et al. [30] state that low expression of p53 ( $\leq 5 \%$ of colored nuclei) and completely negative staining were associated with non-missense mutations (i.e., frameshift, in-frame, nonsense, and splice). Thus, a low or complete lack of immunohistochemical expression may be the result of nonsense mutations leading to the formation of a truncated, non-immunoreactive protein [30-32]. Therefore, immunohistochemical p53 overexpression ( $\geq 70 \%$ of stained nuclei) is a strong surrogate marker of missense gene mutations, but low p53 expression or completely negative immunohistochemical patterns can be responsible for the misclassification of GC groups.

Unlike the TCGA study [8], we found a high percentage of diffuse-type GCs in the "overexpressed p53 and/or HER2+" group (46.5\%). Seventy-nine percent (15/19) of the HER2+ GC cases were of the intestinal type, compared to $33 \%(8 / 24)$ of the HER2- cases. This finding highlights that the p53 evaluation alone (in negative HER2 cases) does not allow us to correctly identify samples corresponding to the TCGA CIN group, and it would explain the high percentage of diffuse-type GCs in the "overexpressed p53 and/or HER2+" group. In addition, the high percentage of diffuse GCs with an overexpressed p53 pattern may be due to the fact that it is also possible to find a strong p53-positive staining without gene alteration [28]. It is clear that without molecular analysis, immunohistochemical p53 staining does not allow us to distinguish p53 wild-type gene samples from mutated ones. The evaluation of cases with low (Fig. 4) or completely lacking p53 immunoexpression is a matter of debate and should be carried out with careful attention, but on a wider case series.

The TCGA study found a high percentage of CDH1 gene mutations in the group of genomically stable GCs [8]. In addition, it has been shown that altered E-cadherin IHC is often not related to molecular CDH1 alterations $[16,17]$, but is associated with a diffuse-type histology $[33,34]$. In fact, we found that cases with aberrant E-cad- 


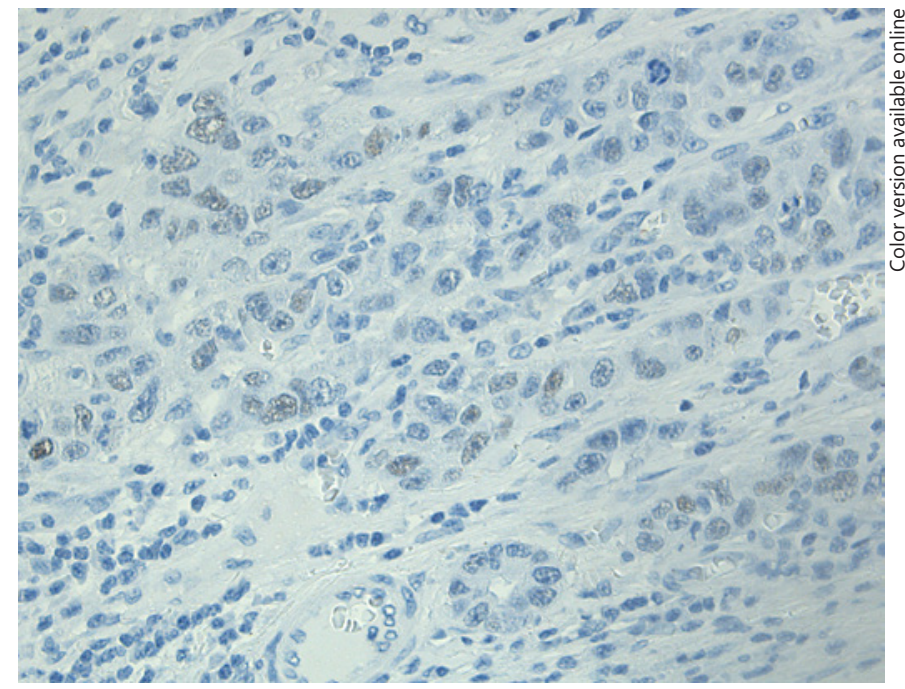

Fig. 4. Scattered p53 staining pattern.

herin were all diffuse-type GCs (Table 2). These tumors were significantly correlated to negative prognostic factors such as a positive $\mathrm{pN}$ status (Table 2).

E-cadherin staining is easily assessable if there is absolute loss of staining (aberrant pattern), or if the staining is only cytoplasmic (aberrant pattern) or membranous (normal pattern). The use of surgical sections allowed a heterogeneous membranous/cytoplasmic pattern (Fig. 5) to be highlighted better, the evaluation of which has not been extensively studied on TMAs. In fact, in most cases there is a heterogeneous staining pattern prevalently cytoplasmic or membranous, and it is not clear how to evaluate these cases. Some studies interpret this heterogeneous staining pattern as normal E-cadherin expression [10], but others as aberrant [35]. To better define this category of GCs, a recent study even suggested considering mesenchymal-like morphologic features as histological criteria, in addition to altered E-cadherin expression [16].

Negative staining for all markers was found in $17.1 \%$ of the GC samples, and we assigned these tumors to the "normal pattern" group. Other studies that performed such an immunophenotypic classification as well identified a group of GCs without altered markers $[10,13]$. It is not clear whether to consider these cases as a separate group or as cases that might have a p53 nonsense mutation.

Through a better understanding of the heterogeneity of immunophenotypic expression markers, the use of surgical samples - rather than solving the critical issue

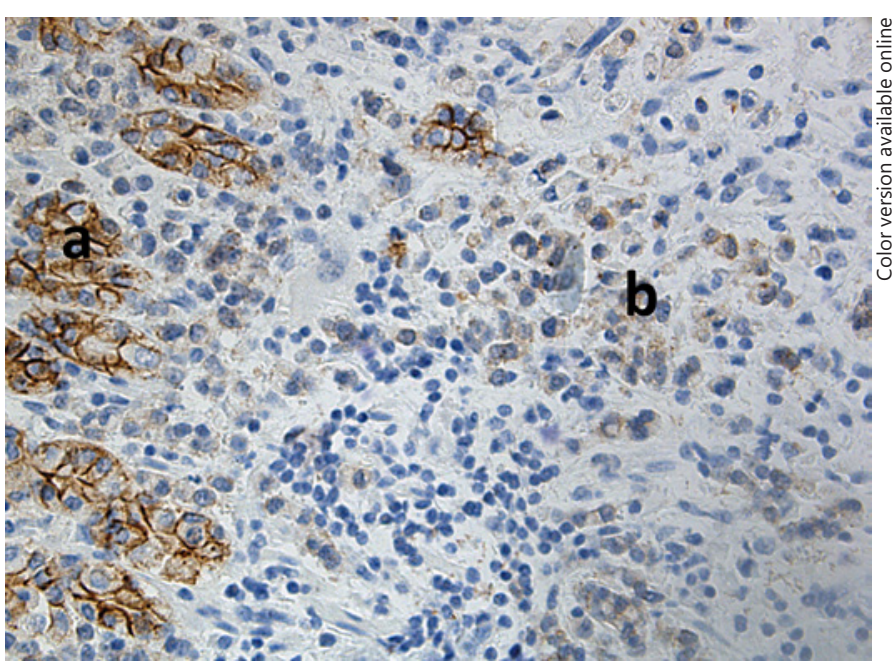

Fig. 5. Heterogeneous (a, membranous; b, cytoplasmic) immunohistochemical E-cadherin pattern in neoplastic gastric cancer cells.

already shown using TMAs - emphasizes even more strongly that some markers are not useful. We can state that the EBV and MMR-D GC categories are well-defined groups of GCs, are mutually exclusive, and are easily assessable by IHC. In addition, these GCs could be good candidates for immunotherapy with PD-1/PD-L1 inhibitors. As regards p53, IHC is not able to define the TGCA CIN group, particularly in HER2-negative cases, because our results highlight a possible misclassification of GCs due to a lack of concordance between immunohistochemical pattern and molecular status. Immunohistochemical E-cadherin assessment needs to be revised, particularly as regards the heterogeneous cytoplasmic/membranous staining pattern, in order to unambiguously identify what group they belong to. The question as to whether to consider the "normal pattern" group as a separate category remains to be clarified.

In conclusion, in our study, only $40 \%$ of GC cases exhibited markers that can serve as an indication for known targeted therapy: 19 HER2+ cases, 5 MMR-D cases, and $4 \mathrm{PD}-\mathrm{L} 1+$ cases (2 EBV+ and 2 overexpressing p53). Furthermore, we suggest that the immunophenotypic classification needs to be reviewed on the basis of a wider case series, especially as regards markers to identify the TGCA CIN and GS groups. These markers should also play a therapeutic role, as PD-L1 does for the EBV and MMR-D groups, and HER2 for the "overexpressed p53 and/or HER2+" group. 


\section{Statement of Ethics}

The present study was approved by the Review Board of the National Institute of Gastroenterology "S. de Bellis," Research Hospital (Castellana Grotte, Bari, Italy), and was conducted in accordance with the Declaration of Helsinki. Prior to enrollment, all participants provided written informed consent.

\section{Disclosure Statement}

The authors have no conflicts of interest to declare.

\section{Funding Sources}

This study was supported by the Italian Ministry of Health (Ricerca Corrente 2018).

\section{Author Contributions}

All authors contributed equally to the work presented in this manuscript.

\section{References}

1 Gullo I, Carneiro F, Oliveira C, Almeida GM. Heterogeneity in gastric cancer: from pure morphology to molecular classifications. Pathobiology. 2018;85(1-2):50-63.

2 Sakuramoto S, Sasako M, Yamaguchi T, Kinoshita T, Fujii M, Nashimoto A, et al. Adjuvant chemotherapy for gastric cancer with S-1, an oral fluoropyrimidine. N Engl J Med. 2007;357(18):1810-20.

3 Cunningham D, Allum WH, Stenning SP, Thompson JN, Van de Velde CJ, Nicolson M, et al. Perioperative chemotherapy versus surgery alone for resectable gastroesophageal cancer. N Engl J Med. 2006;355(1):11-20.

4 Bang YJ, Van Cutsem E, Feyereislova A, Chung HC, Shen L, Sawaki A, et al.; ToGA Trial Investigators. Trastuzumab in combination with chemotherapy versus chemotherapy alone for treatment of HER2-positive advanced gastric or gastro-oesophageal junction cancer (ToGA): a phase 3, open-label, randomised controlled trial. Lancet. 2010;376 (9742):687-97.

5 Fuchs CS, Tomasek J, Yong CJ, Dumitru F, Passalacqua R, Goswami C, et al.; REGARD Trial Investigators. Ramucirumab monotherapy for previously treated advanced gastric or gastro-oesophageal junction adenocarcinoma (REGARD): an international, randomised, multicentre, placebo-controlled, phase 3 trial. Lancet. 2014;383(9911):31-9.

6 Wilke H, Muro K, Van Cutsem E, Oh SC, Bodoky G, Shimada Y, et al.; RAINBOW Study Group. Ramucirumab plus paclitaxel versus placebo plus paclitaxel in patients with previously treated advanced gastric or gastro-oesophageal junction adenocarcinoma (RAINBOW): a double-blind, randomised phase 3 trial. Lancet Oncol. 2014;15(11):1224-35.

$7 \mathrm{Yu} \mathrm{Y}$. Molecular classification and precision therapy of cancer: immune checkpoint inhibitors. Front Med. 2018;12(2):229-35.

8 Bass AJ, Thorsson V, Shmulevich I, Reynolds SM, Miller M, Bernard B, et al.; Cancer Genome Atlas Research Network. Comprehensive molecular characterization of gastric adenocarcinoma. Nature. 2014;513(7517):202-

Immunophenotypic Classification of Gastric Cancer 9.
9 Gonzalez RS, Messing S, Tu X, McMahon LA, Whitney-Miller CL. Immunohistochemistry as a surrogate for molecular subtyping of gastric adenocarcinoma. Hum Pathol. 2016;56: $16-21$.

10 Setia N, Agoston AT, Han HS, Mullen JT, Duda DG, Clark JW, et al. A protein and mRNA expression-based classification of gastric cancer. Mod Pathol. 2016;29(7):772-84.

11 Díaz Del Arco C, Estrada Muñoz L, Molina Roldán E, Cerón Nieto MÁ, Ortega Medina L, García Gómez de Las Heras S, et al. Immunohistochemical classification of gastric cancer based on new molecular biomarkers: a potential predictor of survival. Virchows Arch. 2018;473(6):687-95

12 Birkman EM, Mansuri N, Kurki S, Ålgars A, Lintunen M, Ristamäki R, et al. Gastric cancer: immunohistochemical classification of molecular subtypes and their association with clinicopathological characteristics. Virchows Arch. 2018;472(3):369-82.

13 Ahn S, Lee SJ, Kim Y, Kim A, Shin N, Choi $\mathrm{KU}$, et al. High-throughput protein and mRNA expression-based classification of gastric cancers can identify clinically distinct subtypes, concordant with recent molecular classifications. Am J Surg Pathol. 2017;41(1): 106-15.

14 Ando K, Oki E, Saeki H, Yan Z, Tsuda Y, Hidaka G, et al. Discrimination of p53 immunohistochemistry-positive tumors by its staining pattern in gastric cancer. Cancer Med. 2015;4(1):75-83.

15 Oh HJ, Bae JM, Wen X, Jung S, Kim Y, Kim $\mathrm{KJ}$, et al. p53 expression status is associated with cancer-specific survival in stage III and high-risk stage II colorectal cancer patients treated with oxaliplatin-based adjuvant chemotherapy. Br J Cancer. 2019;120(8):797805.

16 Koh J, Lee KW, Nam SK, Seo AN, Kim JW, Kim JW, et al. Development and validation of an easy-to-implement, practical algorithm for the identification of molecular subtypes of gastric cancer: prognostic and therapeutic implications. Oncologist. 2019;24(12):e1321e1330.
17 Corso G, Carvalho J, Marrelli D, Vindigni C, Carvalho B, Seruca R, et al. Somatic mutations and deletions of the E-cadherin gene predict poor survival of patients with gastric cancer. Clin Oncol. 2013;31(7):868-75.

18 Valentini AM, Di Pinto F, Cariola F, Guerra V, Giannelli G, Caruso ML, et al. PD-L1 expression in colorectal cancer defines three subsets of tumor immune microenvironments. Oncotarget. 2018;9(9):8584-96.

19 Ju X, Shen R, Huang P, Zhai J, Qian X, Wang $\mathrm{Q}$, et al. Predictive relevance of PD-L1 expression with pre-existing TILs in gastric cancer. Oncotarget. 2017;8(59):99372-81.

20 Hofmann M, Stoss O, Shi D, Büttner R, van de Vijver M, Kim W, et al. Assessment of a HER2 scoring system for gastric cancer: results from a validation study. Histopathology. 2008;52(7):797-805.

21 Bartley AN, Washington MK, Colasacco C, Ventura CB, Ismaila N, Benson AB 3rd, et al. HER2 testing and clinical decision making in gastroesophageal adenocarcinoma: guideline from the College of American Pathologists, American Society for Clinical Pathology, and the American Society of Clinical Oncology. J Clin Oncol. 2017;35(4):446-64.

22 Serra O, Galán M, Ginesta MM, Calvo M, Sala $\mathrm{N}$, Salazar R. Comparison and applicability of molecular classifications for gastric cancer. Cancer Treat Rev. 2019;77:29-34.

23 Valentini AM, Di Pinto F, Coletta S, Guerra V, Armentano R, Caruso ML. Tumor microenvironment immune types in gastric cancer are associated with mismatch repair however, not HER2 status. Oncol Lett. 2019;18(2): 1775-85.

24 Lee J, Kim KM. Biomarkers for gastric cancer: molecular classification revisited. Precis $\mathrm{Fu}-$ ture Med. 2017;1:59-68.

25 Palazzo JP, Kafka NJ, Grasso L, Chakrani F, Hanau C, Cuesta $\mathrm{KH}$, et al. The role of p53, $\mathrm{p} 21^{\mathrm{WAF} 1 / \mathrm{ClP} 1}$, and bcl-2 in radioresistant colorectal carcinoma. Hum Pathol. 1997; 28(10):1189-95. 
26 Bell SM, Scott N, Cross D, Sagar P, Lewis FA, Blair GE, et al. Prognostic value of p53 overexpression and $\mathrm{c}-\mathrm{Ki}$-ras gene mutations in colorectal cancer. Gastroenterology. 1993; 104(1):57-64.

27 Momand J, Zambetti GP, Olson DC, George $\mathrm{D}$, Levine AJ. The $m d m-2$ oncogene product forms a complex with the $\mathrm{p} 53$ protein and inhibits p53-mediated transactivation. Cell. 1992;69(7):1237-45.

28 Hall PA, Lane DP. Tumor suppressors: a developing role for p53? Curr Biol. 1997;7(3): R144-7.

29 Cole AJ, Dwight T, Gill AJ, Dickson KA, Zhu $\mathrm{Y}$, Clarkson A, et al. Assessing mutant p53 in primary high-grade serous ovarian cancer using immunohistochemistry and massively parallel sequencing. Sci Rep. 2016;6:26191.
30 Lax SF, Kendall B, Tashiro H, Slebos RJ, Hedrick L. The frequency of p53, K-ras mutations, and microsatellite instability differs in uterine endometrioid and serous carcinoma: evidence of distinct molecular genetic pathways. Cancer. 2000;88:814-24.

31 Shahin MS, Hughes JH, Sood AK, Buller RE. The prognostic significance of p53 tumor suppressor gene alterations in ovarian carcinoma. Cancer. 2000;89:2006-17.
32 Tashiro H, Isacson C, Levine R, Kurman RJ, Cho KR, Hedrick L. p53 gene mutations are common in uterine serous carcinoma and occur early in their pathogenesis. Am J Pathol. 1997; 150:177-85.

33 Gabbert HE, Mueller W, Schneiders A, Meier S, Moll R, Birchmeier W, et al. Prognostic value of E-cadherin expression in 413 gastric carcinomas. Int J Cancer. 1996;69(3):184-9.

34 Blok P, Craanen ME, Dekker W, Tytgat GN. Loss of E-cadherin expression in early gastric cancer. Histopathology. 1999;34(5):410-5.

35 Lazăr D, Tăban S, Ardeleanu C, Dema A, Sporea I, Cornianu M, et al. The immunohistochemical expression of E-cadherin in gastric cancer; correlations with clinicopathological factors and patients' survival. Rom J Morphol Embryol. 2008;49(4):459-67. 